\title{
Robótica Educativa como Herramienta Dirigida al Desarrollo de Pensamiento Algebraico en Edades Tempranas
}

\author{
Educational Robotics as a Tool for the Development of Algebraic Thinking at an Early \\ Age
}

Rocío Damara Merlo-Espino ${ }^{1}$, Vicente Rodríguez-Hernández ${ }^{2}$ y Víctor Manuel Castaño-Meneses ${ }^{3}$

\section{() EDICIÓN: \#E-CIVTAC}

Recibido: 31/julio/2020

Aceptado: 6/septiembre/2020

Publicado: 25/septiembre/2020

'México

${ }^{2}$ México

${ }^{3}$ México

\section{IIIInstitución}

1Universidad Autónoma de Querétaro 2Universidad Autónoma de Querétaro ${ }^{3}$ Centro de Física Aplicada y Tecnología Avanzada

\section{Correo Eletrónico}

1rocio.damara.merlo@uaq.mx vicente.rodriguez@uaq.mx 3meneses@unam.mx

\section{(D) ORCID}

${ }^{1}$ https://orcid.org/0000-0002-3642-1347 ${ }^{2} \mathrm{https} / / /$ orcid.org/0000-0001-5113-965X 3https://orcid.org/0000-0002-2983-5293

\section{Citar así: GCAPA / IEEE}

Merlo-Espino, R., Rodríguez-Hernández, V. \& Castaño-Meneses, V. (2020) Robótica Educativa como Herramienta Dirigida al Desarrollo de Pensamiento Algebraico en Edades Tempranas. Revista Tecnológica-Educativa Docentes 2.0, 9(2), 245-253.

https://doi.org/10.37843/rted.v9i2.170

R. Merlo-Espino, V. Rodríguez-Hernández y V. Castaño-Meneses, "Robótica Educativa como Herramienta Dirigida al Desarrollo de Pensamiento Algebraico en Edades Tempranas", RTED, vol. 9, n. ${ }^{\circ}$ 2, pp. 245-253, sep. 2020 .

\section{Resumen}

Desarrollar pensamiento algebraico propicia construir ideas más complejas, necesarias para la formación de ciudadanos críticos, con argumentos fundamentados. Tradicionalmente la escuela siempre ha formado en competencias matemáticas, no obstante, los resultados no han favorecido a la mayoría de los ciudadanos. Los planes educativos en México introducen temas algebraicos, formalmente hasta educación secundaria, postergando la formación temprana muchos años. El objetivo general fue explorar robótica educativa como recurso didáctico dirigido a desarrollar pensamiento algebraico con estudiantes en edades tempranas. Se instrumentó una metodología mixta en estudio de caso a cinco estudiantes con edad de 7 a 9 años, con un taller perteneciente a la Universidad Autónoma de Querétaro, México. La propuesta implemento una secuencia didáctica sustentada en situaciones problema con ensambles de robots, enlazando el tema engranes, en conjunto con proporcionalidad directa. El hallazgo más importante fue identificar a la robótica educativa como herramienta potencial para favorecer la adquisición a conceptos algebraicos de incógnita y variable en una relación funcional. Finalmente, robótica educativa se posiciona como un recurso didáctico poderoso del siglo XXI, además de conjuntar diversas ciencias, posibilita trabajar contenidos académicos fuera del aula, favoreciendo el desarrollo cognitivo.

Palabras clave: Robótica educativa, pensamiento algebraico, proporcionalidad directa, secuencia didáctica.

\begin{abstract}
Developing algebraic thinking encourages the construction of more complex ideas, necessary for the formation of critical citizens, with well-founded arguments. Traditionally, the school has always trained in mathematical skills, however, the results have not favored most citizens. Educational plans in Mexico introduce algebraic topics, formally until secondary education, postponing early training for many years. The general objective was to explore educational robotics as a didactic resource aimed at developing algebraic thinking with students at an early age. A mixed methodology was implemented in a case study of five students aged 7 to 9 years, with a workshop belonging to the Autonomous University of Querétaro, Mexico. The proposal implemented a didactic sequence based on problem situations with robot assemblies, linking the subject of gears, together with direct proportionality. The most important finding was to identify educational robotics as a potential tool to favor the acquisition of algebraic concepts of unknown and variable in a functional relationship. Finally, educational robotics is positioned as a powerful didactic resource of the XXI century, in addition to combining various sciences, it makes it possible to work academic content outside the classroom, favoring cognitive development.
\end{abstract}

Keywords: Educational robotics, algebraic thinking, direct proportionality, didactic sequence. 


\section{Introducción}

En el presente trabajo de investigación se documentan los resultados de una situación didáctica la cual implementa robótica educativa como herramienta dirigida a desarrollar pensamiento algebraico a través del tema de proporcionalidad directa. Dicha investigación surge sustentada en la necesidad detectada en un taller de estudiantes multigrado de edades tempranas, implementado en la Universidad Autónoma de Querétaro, México. En dicho taller, se enseñan temas de ciencia, tecnología, ingeniería y matemáticas conocidas como CTIM, conjuntadas en la construcción de robots.

Al trabajar en el taller con los contenidos antes enunciados, los estudiantes tienen que realizar equivalencias y cálculos, los cuales permiten seleccionar combinaciones de engranes, adecuadas en el ensamble de un robot. Por ser conocimientos complejos, aunado a que su consolidación se da a lo largo de la escolarización, sería importante propiciar un análisis más profundo enlazando el tema de proporcionalidad directa, fundamental en la comprensión de un sistema de engranes.

Por lo anterior, se presenta la experiencia obtenida en el primer pilotaje realizado al implementar una secuencia didáctica abordando el problema anterior, la secuencia contiene cinco situaciones problema, que apoyan la enseñanza de engranes con estudiantes de 7 a 9 años, incorporando robótica como eje rector de la intervención. La experiencia se estructura como artículo, desarrollándose en cuatro grandes apartados: 1) Desarrollo del problema y fundamentos teóricos., 2) Metodología implementada., 3) Resultados obtenidos., 4) Conclusiones y hallazgos relevantes

Para finalizar, es relevante enunciar el aporte de la perspectiva interdisciplinaria plasmada en la investigación, la cual se materializa inicialmente en el diseño de la secuencia didáctica que tiene como finalidad potencializar a través de robótica educativa el pensamiento algebraico por la ruta de la proporcionalidad directa. Esta última, como parte de la aritmética introduce a conceptos algebraicos como incógnita y variable en una relación funcional, importantes para el pensamiento algebraico.

\section{Desarrollo}

Dentro del taller de robótica los estudiantes resuelven situaciones problema, rebasando frecuentemente el grado de conocimiento conceptual requerido para profundizar en temas de robótica. En clase los temas comúnmente revisados corresponden a áreas de Ciencia, Tecnología, Ingeniería y Matemáticas conocidas por su acrónimo CTIM (Chalmers, 2018). En el caso específico de esta investigación, el interés versó sobre el área de matemáticas, en el taller de robótica uno de los principales problemas detectados es cuando trabajan el tema de engranes, los estudiantes en su mayoría no han tenido acercamiento a conceptos como funciones, relacionadas directamente con el álgebra.

En el mismo sentido, los estudiantes participantes en el taller de robótica poseen algunas habilidades relacionadas a la aritmética, saben sumar, restar, multiplicar y dividir. El grado de dominio en ese tipo de operaciones matemáticas varían con relación a edad, experiencia utilizando operaciones matemáticas, rango numérico que manejen, por mencionar algunas (Broitman, 2000). No obstante, aunque posean conocimientos previos para resolver problemas, muchas veces no es suficiente, debido al nivel de comprensión requerido al ensamblar robots.

De este modo, enseñar el tema de engranes, indiscutiblemente presenta la necesidad de vincular el tema de proporcionalidad directa, requiriendo comprensión en la relación de las magnitudes distintas, puestas en juego al buscar una equivalencia. En este sentido, en los engranes existe una variable $\mathrm{X}$ $\mathrm{y}$ una variable $\mathrm{Y}$, estas tienen una relación directamente proporcional, si una aumenta o disminuye, la otra también. Considerando lo anterior, se observan dificultades cuando los estudiantes seleccionan medidas de engranes para dar fuerza, velocidad o dirección a su robot, teniendo como consecuencia escasa comprensión en los problemas a resolver, así como una carente apropiación de conocimiento.

Ante las necesidades enunciadas en el taller de robótica, se formula la siguiente pregunta: ¿Cómo implementar robótica educativa con la finalidad de potencializar el pensamiento algebraico, logrando así mejorar estrategias de resolución de problemas al armar un robot? Por lo anterior, se propuso como objetivo general, diseñar e implementar una secuencia didáctica que implementó robótica educativa como recurso didáctico con estudiantes en edades tempranas.

\section{Fundamentos Teóricos}


La tecnología digital asociada al ámbito educativo se conceptualiza principalmente de dos maneras: 1) Tecnología como medio de información, implicando recibir o trasmitir datos, sin necesidad de generar un aprendizaje a largo plazo. 2) Tecnología como medio de construcción, implicando que los estudiantes se involucren en el proceso de enseñanzaaprendizaje, apropiándose del conocimiento o habilidades esperadas (Papert \& Harel, 2002).

En este sentido, la robótica educativa es posicionada como tecnología para construir conocimiento, caracterizada por dos atributos: 1) Tiene virtud de objeto a fin de contribuir en pensar sobre nuevos aprendizajes adquiridos, el estudiante la utiliza como artefacto cognitivo, convirtiéndose así en una parte constitutiva en la construcción del conocimiento. 2) Colabora con las construcciones o representaciones realizadas de manera física, auditiva, visual de los conceptos de aprendizaje. Otorgando la posibilidad de involucrar y concientizar al estudiante durante su proceso de aprendizaje (Ruiz-Velasco et al., 2010).

Existen trabajos previos los cuales documentan algunos beneficios al introducir robótica educativa en educación básica como medio o herramienta para la socialización del aprendizaje, adquisición de nuevos conocimientos, incluyendo el desarrollo a esquemas de pensamiento. Así pues, cuando los estudiantes interactúan con la herramienta, se favorece el aprendizaje significativo, potencializando además el desarrollo de estrategias en conjunto a los métodos de planificación del propio aprendizaje (Di Lieto et al., 2017; Mataric, 2004; Vargas et al., 2019).

En relación con la definición encontramos diversas concepciones, en este sentido Ruiz-Velasco et al., (2010) la conceptualizan como una disciplina la cual permite desarrollar robots en el ámbito educativo, sirviendo como primera experiencia cognitiva de los alumnos sobre tecnología o ciencias. Asimismo, es una herramienta con mucho potencial en la construcción de conocimientos disciplinares, competencias y alfabetizaciones, dentro del rubro de tecnologías educativas. Razones que la postulan como idónea hacia la alfabetización matemática, en estudiantes de cualquier edad.

Por otro lado, la Organización para la Cooperación y el Desarrollo Económico OCDE (2017), plantea a la alfabetización o competencia matemática, más allá de la adquisición de conceptos, datos o procedimientos. Implicaría una serie de habilidades, así como el desarrollo de procesos cognitivos que el estudiante consolidará a lo largo de su vida. Lo anterior, propiciaría estudiantes con pensamiento crítico en todas las áreas, capaces de tomar decisiones fundamentadas.

La competencia matemática examinada en el Programa para la Evaluación Internacional de Alumnos, por sus siglas PISA, implica lo siguiente: "Capacidad del individuo encaminada a formular, emplear e interpretar las matemáticas en distintos contextos. Incluye razonar matemáticamente, utilizar conceptos, procedimientos, herramientas, así como hechos matemáticos. Permitiendo juicios $\mathrm{y}$ decisiones fundamentados que necesitan los ciudadanos constructivos, comprometidos en reflexión constante (OCDE, 2017, p. 64)".

Considerando lo anterior, sería oportuno intervenir desde edades tempranas para el desarrollo de habilidades de pensamiento que permitan construir la competencia matemática. En este sentido, existen experiencias previas sostenidas en la posibilidad de trabajar conceptos como proporcionalidad directa a través de problemas de estructura multiplicativa, las cuales plantean resolución vía suma, resta, multiplicación o división (Block et al., 1997, 2015; Mochón-Cohen, 2012).

En relación con lo anterior, los planes y programas educativos de la Secretaría de Educación Pública SEP (2011), en nivel de Educación Básica correspondiente a primaria, marcan iniciar el estudio de la proporcionalidad principalmente en los dos últimos grados, quinto o sexto. Es hasta estos grados, se plantea desarrollar pensamiento proporcional comenzando a introducir problemas de estructura multiplicativa: multiplicación, división, número racional, escala, porcentaje, en conjunto con la probabilidad.

Sin embargo, de acuerdo a la descripción de la competencia matemática planteada por la SEP (2011), un estudiante además de desarrollar, tendría que consolidar a lo largo de la trayectoria escolar lo siguiente: 1) Resolver problemas de manera autónoma. 2) Comunicar información matemática. 3) Validar procedimientos y resultados. 4) Manejar técnicas eficientemente. Lo anterior no es una tarea sencilla, por lo cual sería relevante analizar a detalle en qué medida se logra, debido a que los indicadores nacionales e internacionales en la alfabetización matemática, indicarían lo contrario (OCDE, 2019). A continuación, se presenta la Tabla 1, representando 
las cuatro competencias matemáticas para desarrollar en los estudiantes al término de la Educación Básica en México.

\section{Tabla 1}

Competencias Matemáticas Para Desarrollar en Educación Básica.

\begin{tabular}{|c|c|c|c|}
\hline $\begin{array}{c}\text { Resolver } \\
\text { problemas de } \\
\text { manera } \\
\text { autónoma }\end{array}$ & $\begin{array}{c}\text { Comunicar } \\
\text { información } \\
\text { matemática }\end{array}$ & $\begin{array}{c}\text { Validar } \\
\text { procedimientos } \\
\text { y resultados }\end{array}$ & $\begin{array}{c}\text { Manejar } \\
\text { técnicas } \\
\text { eficientemente }\end{array}$ \\
\hline $\begin{array}{l}\text { Identifica, } \\
\text { plantea y } \\
\text { resuelve } \\
\text { diferentes tipos } \\
\text { de problemas o } \\
\text { situaciones }\end{array}$ & $\begin{array}{l}\text { Expresa, } \\
\text { representa e } \\
\text { interpreta } \\
\text { información } \\
\text { matemática }\end{array}$ & $\begin{array}{l}\text { Adquiere } \\
\text { confianza } \\
\text { suficiente para } \\
\text { explicar y } \\
\text { justificar los } \\
\text { procedimientos }\end{array}$ & $\begin{array}{l}\text { Usa } \\
\text { procedimientos } \\
\text { y formas de } \\
\text { representación } \\
\text { a efectuar } \\
\text { cálculos }\end{array}$ \\
\hline $\begin{array}{l}\text { Resuelve un } \\
\text { problema } \\
\text { utilizando } \\
\text { diversos } \\
\text { procedimientos }\end{array}$ & $\begin{array}{l}\text { Comprende y } \\
\text { emplea } \\
\text { diferentes } \\
\text { formas de } \\
\text { representar la } \\
\text { información }\end{array}$ & $\begin{array}{l}\text { y soluciones } \\
\text { encontradas } \\
\text { Argumenta a } \\
\text { sus } \\
\text { posibilidades }\end{array}$ & $\begin{array}{l}\text { Desarrolla el } \\
\text { significado y } \\
\text { uso d ellos } \\
\text { números y las } \\
\text { operaciones }\end{array}$ \\
\hline $\begin{array}{l}\text { Comprueba la } \\
\text { eficacia de un } \\
\text { procedimiento }\end{array}$ & $\begin{array}{l}\text { Expone con } \\
\text { claridad las } \\
\text { ideas }\end{array}$ & $\begin{array}{l}\text { Usa } \\
\text { razonamiento } \\
\text { deductivo y } \\
\text { demostración } \\
\text { formal }\end{array}$ & $\begin{array}{l}\text { Elige } \\
\text { adecuadamente } \\
\text { las operaciones } \\
\text { al resolver un } \\
\text { problema }\end{array}$ \\
\hline \multirow{2}{*}{$\begin{array}{l}\text { Generaliza } \\
\text { procedimientos } \\
\text { de resolución }\end{array}$} & $\begin{array}{l}\text { propiedades, } \\
\text { características } \\
\text { o tendencias }\end{array}$ & & $\begin{array}{l}\text { Utiliza el } \\
\text { cálculo mental } \\
\text { y la estimación }\end{array}$ \\
\hline & & & $\begin{array}{l}\text { Evalúa la } \\
\text { pertinencia de } \\
\text { los resultados. }\end{array}$ \\
\hline
\end{tabular}

Nota. con datos de (SEP, 2011). Esta tabla describe las cuatro competencias matemáticas y las habilidades que las componen, elaboración propia (2020).

Considerando lo anterior, se propone en la investigación implementar una secuencia didáctica, con el propósito de profundizar contenidos de la clase de robótica, específicamente a través de la proporcionalidad directa. Si bien, el material didáctico utilizado funciona para trabajar contenidos de manera concreta, posibilitando la observación e interacción con el objeto de aprendizaje, no es suficiente cuando se pretende consolidar contenidos del área matemática (Fregona, 2013).

En este sentido, el diseño de la secuencia implementada considera variables didácticas, las cuales contemplan determinados elementos que el profesor modifica intencionalmente, para provocar un cambio de estrategia de los alumnos, con la finalidad de llegar a un saber determinado (Brousseau, 2000).
Las variables didácticas seleccionadas para la investigación consideraron principalmente el nivel de conocimiento tanto en matemáticas como en robótica de los estudiantes participantes. Por otra parte, también se consideró el nivel de desarrollo cognitivo de los estudiantes, así como el tipo de problema a resolver, planteado en cada situación.

Teniendo en cuenta las variables didácticas, en la generalidad intervenciones en matemáticas, consideran el tipo de problema, así como el lugar que ocupará la incógnita a resolver. Siendo relevante para la selección de lo anterior, observar en una previa evaluación las estrategias de resolución presentadas en los estudiantes al tomar decisiones. Lo anterior, otorga conocimiento, de en qué medida y cómo el docente ofrecerá situaciones dirigidas al progreso en la resolución de problemas, con la finalidad de mejorar la alfabetización matemática (EcheverríaAnaya, 2019; Torres-Chávez, 2019).

A continuación, se presenta el diseño de la secuencia didáctica implementada, dicha secuencia describe el objetivo y las principales variables didácticas consideradas para la intervención, ver Tabla 2.

\section{Tabla 2}

Diseño de Secuencia Didáctica Implementado en la Clase Multigrado de Robótica.

\begin{tabular}{|c|c|c|}
\hline Instrumento & Objetivo & $\begin{array}{l}\text { Variables } \\
\text { didácticas }\end{array}$ \\
\hline $\begin{array}{l}\text { Secuencia } \\
\text { didáctica }\end{array}$ & $\begin{array}{l}\text { Desarrollar } \\
\text { pensamiento } \\
\text { algebraico por } \\
\text { medio de problemas } \\
\text { de equivalencia en el }\end{array}$ & $\begin{array}{l}\text { Elaboración de } 5 \\
\text { situaciones } \\
\text { Implementaciones } \\
\text { durante } 5 \text { dias } \\
\text { Duración } 45 \text { minutos }\end{array}$ \\
\hline & $\begin{array}{l}\text { uso de engranes, } \\
\text { utilizando tablas de } \\
\text { proporcionalidad }\end{array}$ & $\begin{array}{l}\text { a } 1 \text { hora cada } \\
\text { situación }\end{array}$ \\
\hline
\end{tabular}

Material didáctico Set 9686, Maquinas Simples $\mathrm{y}$ Motorizadas, LEGO Educación Hoja de papel con problemas impresos

Problemas de estructura multiplicativa (proporcionalidad) Uso de números naturales

Rango numérico del 1 al 100

Nota. Esta tabla describe de manera general los elementos considerados para el diseño de la secuencia didáctica en la clase de robótica, elaboración propia (2020). 


\section{Metodología}

El enfoque de la presente investigación fue mixto, reuniendo las virtudes de lo cuantitativocualitativo, en este sentido Hernández, Fernández y Baptista (2010), consideran que dicho enfoque posibilita realizar análisis de datos tanto descriptiva como numéricamente, ajustándose a necesidades requeridas en la investigación. El diseño de investigación fue no experimental, transeccional, correlacional-causal, pretendiendo recolectar datos en un tiempo específico, con el propósito de describir variables, analizar su incidencia, así como la relación entre estas (Hernández et al., 2010).

Por otra parte, se utilizó estudio de caso como método de investigación, realizando intervención a un grupo multigrado de 15 estudiantes. Respecto al pilotaje de la secuencia didáctica se selecciona una muestra por conveniencia de cinco estudiantes, hombres de 7 a 9 años de edad. La investigación fue implementada en la Universidad Autónoma de Querétaro, Facultad de Ingeniería, perteneciente a la ciudad de Santiago de Querétaro, México. La Tabla 3 muestra los instrumentos implementados en la recolección de información.

Tabla 3

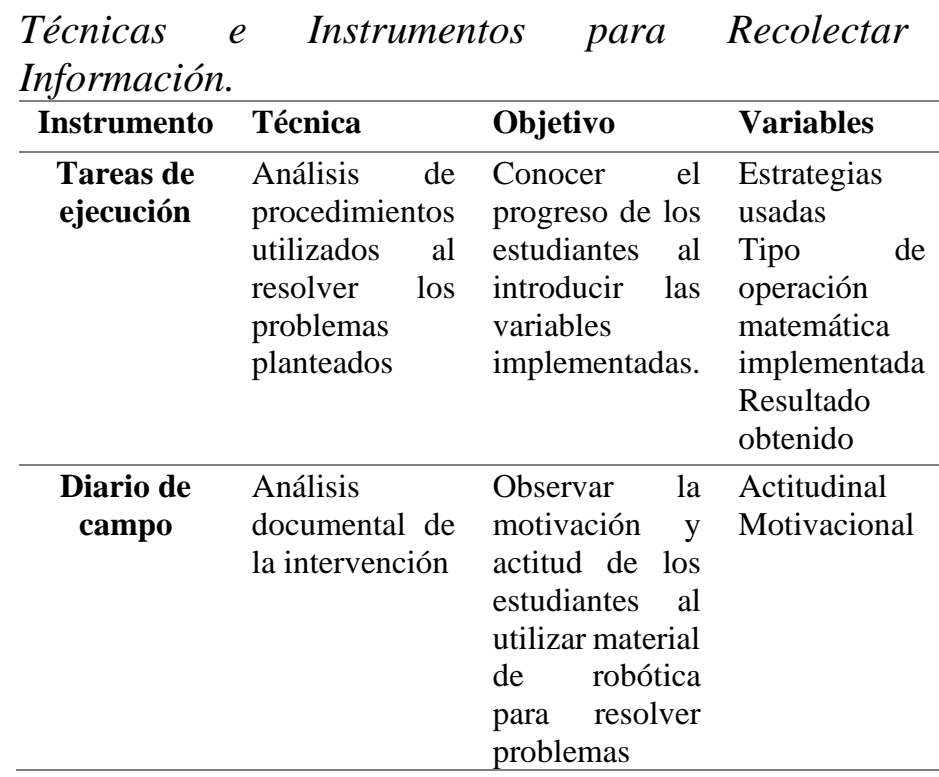

Nota. Esta tabla describe los instrumentos y técnicas implementados en la recolección de datos durante la intervención realizada. Se describen dos instrumentos, con sus técnicas, objetivos y variables evaluadas, elaboración propia (2020).

\section{Instrumento 1, tareas de ejecución}

El instrumento recolecta datos en relación con tres aspectos: 1) estrategias usadas, 2) tipo de operación matemática implementada, 3) resultado obtenido. Los tres aspectos representan las variables medidas, teniendo como objetivo conocer el progreso de los estudiantes durante la secuencia didáctica aplicada. Para implementar la secuencia se utilizaron hojas de papel impresas que incluyeron instrucciones, problemas, preguntas y tablas de proporcionalidad directa.

La primera variable indagó las estrategias usadas al resolver los problemas planteados, de acuerdo con la edad de los estudiantes se contemplaron las siguientes posibles estrategias: representaciones gráficas, procedimientos de conteo, operaciones matemáticas y resolución en lo concreto a través de material didáctico. De las estrategias enunciadas resaltan solo tres tipos, a continuación, se describen en orden de complejidad.

a) Estrategia dibujo, implica la representación gráfica del problema, optan por dibujar para organizar las ideas, favoreciendo algunas veces la compresión del problema mismo. Lo anterior sucede cuando el estudiante no tiene una operación matemática en mente o interiorizada, así como cuando tampoco han tenido experiencia previa al incorporar material didáctico que ayude a resolver.

b) Estrategia uso material didáctico, implica incorporar material de robótica permitiendo hacer conteo directo $\mathrm{o}$ estimaciones, permitiendo observar los tamaños de engrane. El conteo directo se observaría cuando el estudiante agarre los engranes en físico, procediendo a contar cada diente en éstos, así como la relación de vueltas que dan cada uno en función del otro. Por ejemplo, un engrane con 4 dientes, dará dos vueltas completas, compensando una vuelta completa a un engrane con 8 dientes.

c) Estrategia operación matemática, teniendo como objetivo analizar la utilización de algoritmos de suma, resta, multiplicación y división. Es importante enunciar que esta estrategia, implicaría 
d) ser la más evolucionada comparando con las pasadas dos.

La segunda variable analizada es la implementación de operaciones matemáticas, en este sentido se registró el tipo de operación aritmética seleccionada. La utilización de cualquiera denotará, la comprensión del problema, así como los conocimientos previos puestos en marcha para resolver el problema. Los problemas implicaron realizar tablas de proporción directa, pudiendo utilizar como opción de operación matemática: a) suma, b) resta, c) multiplicación, d) división.

La tercera variable analiza las respuestas dadas por cada estudiante, clasificándolas como acertadas o no acertadas, teniendo como objetivo un análisis estadístico en función de errores o aciertos presentados en cada estudiante durante toda la intervención. Los errores presentados por los alumnos servirán solo para una mejor comprensión del fenómeno de investigación, no siendo considerados como única evidencia del aprendizaje. No obstante, lo anterior ayudará en la valoración al progreso de los estudiantes, así como para ajustes futuros de la secuencia didáctica.

\section{Instrumento 2, Diario de Campo}

Durante las 5 sesiones se registró la motivación como variable cualitativa a considerar como parte importante del proceso de enseñanza-aprendizaje, las observaciones documentaron lo siguiente: 1) El entusiasmo expresado al resolver problemas que enlazarán el tema de robótica, así como el uso de material didáctico. 2) Aspecto actitudinales, observando el comportamiento de los estudiantes al resolver los retos presentados. 3) Interacciones entre compañeros, con especial interés en el trabajo colaborativo y la comunicación.

\section{Resultados}

Los resultados recabados son tanto cuantitativos como cualitativos, son presentados con relación a los instrumentos descritos en párrafos anteriores: 1) Tareas de ejecución, implicó análisis de procedimientos utilizados por los estudiantes al resolver los problemas. 2) Diario de campo, recabó información cualitativa durante la implementación de la secuencia.

\section{Tareas de Ejecución}

Variable 1, Estrategias utilizadas para resolver las situaciones problema.

Las estrategias observadas son tres: dibujo, material didáctico y operación matemática. Primera situación: Dibujo, 1 estudiante., Material didáctico, 3 estudiantes., Operación matemática, 1 estudiante. Segunda situación: Material didáctico, 5 estudiantes., Operación matemática, 4 estudiantes. Tercera situación: Material didáctico, 5 estudiantes., Operación matemática, 5 estudiantes. Cuarta situación: Material didáctico, 4 estudiantes., Operación matemática, 5 estudiantes. Quinta situación: Material didáctico, 3 estudiantes., Operación matemática, 5 estudiantes.

La intervención fue solo a 5 estudiantes, encontrando relevante la utilización de dibujo como estrategia de resolución por parte de un estudiante. Dicha estrategia no vuelve a presentarse durante las demás sesiones, mostrando un gran avance en la evolución de sus estrategias. Por otra parte, es interesante encontrar más de una estrategia seleccionada por algunos estudiantes al resolver los problemas, presuponiéndose lo anterior como parte de la comprobación de los resultados. A continuación, es presentada la Figura 1, representando gráficamente los datos anteriores.

\section{Figura 1}

Estrategias Utilizadas para Resolver las Situaciones Problema.

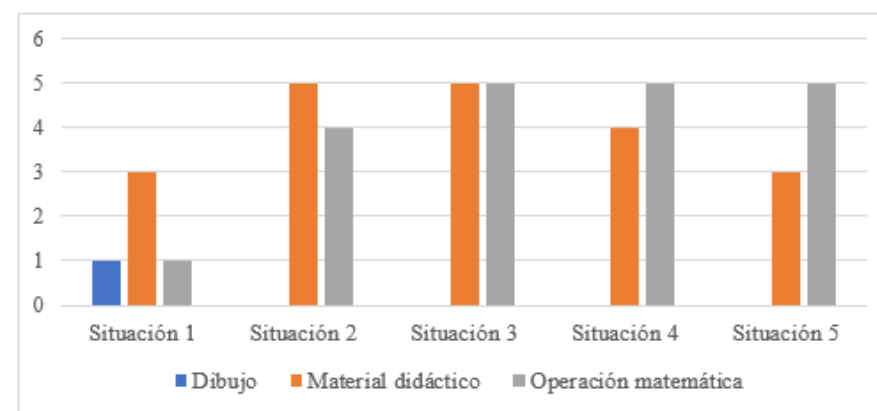

Nota. La figura describe las estrategias de resolución utilizadas por los estudiantes en cada situación, se observa el uso de una o más estrategias para una misma situación problema, elaboración propia (2020).

Variable 2, Tipo de operación matemática implementada. Los problemas implicaban realizar tablas de proporción directa, pudiendo utilizar como opción: suma, resta, multiplicación y división. Primera situación: Suma, 2 estudiantes., 
Multiplicación, 3 estudiantes. Segunda situación: Suma, 1 estudiante., Multiplicación, 4 estudiantes. Tercera situación: Multiplicación, 5 estudiantes. Cuarta situación: Multiplicación, 5 estudiantes. Quinta situación: Multiplicación, 4 estudiantes., División, 1 estudiante, la resta como opción de respuesta, es contemplada como posible respuesta, sin embargo, no se presenta en quinta situación. Por otra parte, en la implementación de la situación 3 y 4, los alumnos seleccionan misma opción de operación. Por último, se encuentra solo a un estudiante en la última sesión implementando el uso de la división. A continuación, es presentada la Figura 2, representando gráficamente los datos anteriores.

\section{Figura 2}

Tipo de Operación Matemática Implementada.

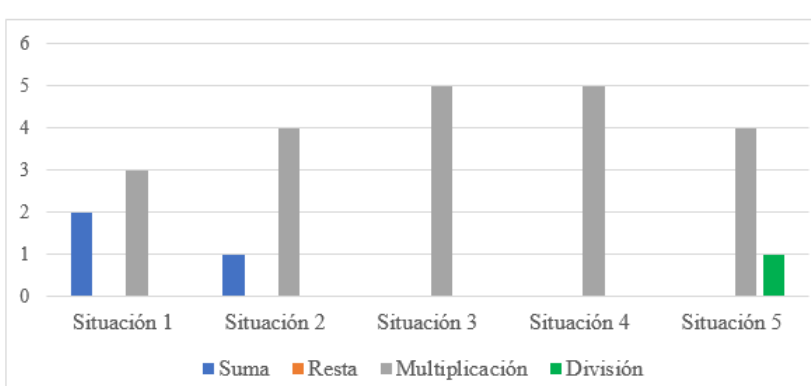

Nota. La figura describe las operaciones matemáticas usadas por los estudiantes en cada situación problema. Es importante señalar que se esperaban principalmente 4 tipos de operación en la resolución: suma, resta, multiplicación y división, elaboración propia (2020).

Variable 3, Resultados obtenidos en cada situación problema. Los resultados son analizados en función de las respuestas escritas otorgadas a cada problema, clasificándolas para un mejor procesamiento como respuestas acertadas o no acertadas. Lo anterior enunciado solo es considerado para comparar estadística y gráficamente todas las situaciones, sirviendo como valoración de la secuencia implementada. En la primera y segunda situación un estudiante presenta error y los demás no. En situación tercera, cuarta y quinta no se encuentran errores en los resultados finales. No obstante, durante la situación tercera se observa a tres estudiantes modificando estrategias de resolución y resultados, sucediendo después de intercambiar información con sus compañeros. A continuación, es presentada la Figura 3, representando gráficamente los datos anteriores.

\section{Figura 3}

Resultados Obtenidos en Cada Situación Problema.

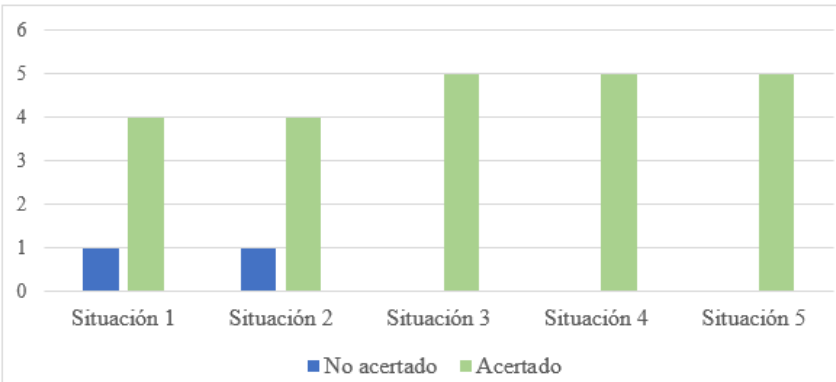

Nota. La figura describe el resultado final dado por los estudiantes en cada situación problema, presentándose como acertado o no. Sin embargo, lo relevante fue documentar el proceso de evolución de las estrategias de resolución por cada estudiante, elaboración propia (2020).

\section{Diario de Campo}

La motivación como variable cualitativa es observada en tres aspectos:1) La clase de robótica educativa en si misma causa motivación, la asistencia a clase es voluntaria. 2) Incorporar problemas reales con temática de robots también causa interés en los estudiantes, mostrando entusiasmo al ensamblar y resolver los retos presentados. 3) Trabajar con material didáctico de robótica como apoyo para resolver retos, genera una mayor confianza al resolver, posibilitando comprobación inmediata de la solución seleccionada por los estudiantes.

En el mismo sentido, la actitud de los estudiantes durante las clases da evidencia de motivación. La conducta general mostrada fue tranquila, cooperativa, con interacción y comunicación constante. No obstante, es importante señalar que durante la tercera situación decayó el ánimo de un estudiante durante clase. Mostrando una atención dispersa a las instrucciones, así como en las actividades encomendadas. El origen de la conducta apática detectada en el estudiante es desconocido. Sin embargo, puede presuponerse que se debió a causas relacionadas al contexto familiar del alumno, ya que no se presentó en otras intervenciones.

A continuación, es presentada la Figura 4, representado de manera gráfica la motivación constante en toda la intervención 


\section{Figura 4}

\section{Motivación Presentada por los Estudiantes.}

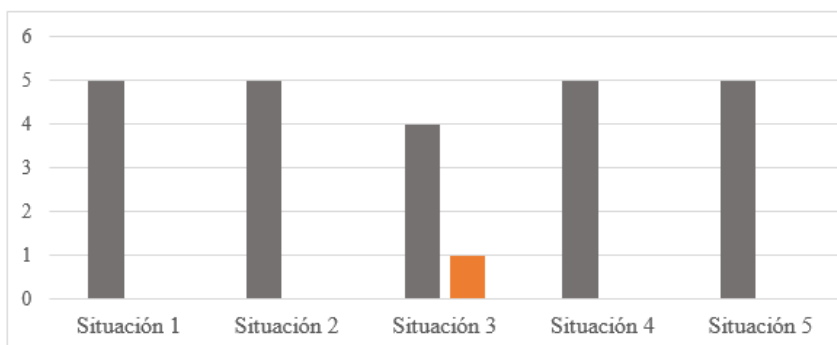

- Motivado No motivado

Nota. La figura describe la motivación presentada por los estudiantes en cada situación problema, la cual se mantiene constante y alta en la mayoría de las intervenciones, elaboración propia (2020).

\section{Conclusiones}

Frente a la evidencia recabada en investigaciones previas consultadas, se detecta la implementación de robótica educativa desde hace algunos años con diversas poblaciones y variados contextos educativos. Encontrando que, gracias a su dinamismo, accesibilidad y versatilidad, como características distintivas a ésta, posibilita trabajar contenidos curriculares en contextos educativos formales, no formales e informales. Así, se destacan las ventajas al usarla en ámbitos de enseñanzaaprendizaje, específicamente para el área de Ciencia, Tecnología, Ingeniería y Matemáticas, conocidas como CTIM o STEM. Por lo anterior, es importante conocer que la robótica educativa se puede implementar como metodología, material didáctico, recurso educativo, por mencionar algunos, considerando su uso con relación a las necesidades del contexto educativo.

En relación con la investigación realizada del taller no escolarizado multigrado de robótica, se propone a la robótica educativa como alternativa didáctica que potencializa el pensamiento algebraico. Respecto a lo anterior, cuando los estudiantes de edades tempranas aún están consolidando conocimientos y habilidades en lo concreto, necesitan apoyarse de objetos físicos para pensar, los cuales permitirán operar con y en la realidad. Más aún, cuando las actividades implican encontrar relaciones funcionales entre magnitudes, trabajo requerido seleccionan engranes y ensamblan robots.

En este sentido, algunos estudiantes generalmente no tienen apropiado el concepto proporcionalidad directa, teniendo errores con los robots ensamblados. Lo anterior, como consecuencia de no identificar relaciones proporcionales, requeridas en conceptos como fuerza, velocidad o dirección a sus robots. No obstante, al aprovechar la problemática referida, la secuencia didáctica diseñada e implementada, muestra resultados preliminares favorables, encontrando una notable mejoría con estrategias implicadas para comprender y resolver problemas que requieren selección de engranes.

Por lo anterior, la experiencia es valorada positivamente como primer acercamiento al problema detectado, algunos hallazgos preliminares relevantes son: 1) Plantear problemas reales, contextualizados y desafiantes, idóneos con retos a robots que involucran, rapidez, fuerza u velocidad. 2) Utilizar tablas de relación proporcional directa, otorgándolas impresas, buscando propiciar un análisis profundo al área matemática, con la finalidad de obtener procedimientos diversos en las respuestas. 3) Proporcionar material didáctico que les permita operar en lo concreto cuando requieran observar proporcionalidad de los engranes en los robots ensamblados, oportuno con estudiantes operadores concretos.

No obstante, aunque ya existe lo anterior enunciado se coloca como propuesta diseñar secuencias didácticas considerando el uso de tecnología, colocando énfasis a la adquisición conceptual significativa, en este caso el área matemática. Asimismo, estimar los saberes previos, en conjunto de las habilidades mentales que poseen los estudiantes, con relación a la etapa cognitiva detectada, es fundamental para poder evolucionar sus estrategias en resolución de problemas, así como también consolidar la alfabetización matemática.

Finalmente, la robótica educativa se posiciona como un recurso didáctico muy pertinente en el siglo XXI, posibilitando trabajar contenidos, competencias y habilidades a estudiantes de diferentes niveles educativos. Por otra parte, es relevante el desarrollo profesional docente desde la perspectiva inter, multi y transdisciplinaria, requerida para intervenciones de mejor calidad en la actualidad. En definitiva, experiencias pasadas muestran que implementar solo tecnología con estudiantes no genera impacto trascendental a la educación, pero conjuntada con otras áreas como: psicología, pedagogía y neurociencias, podría brindar interesantes resultados aplicados al campo educativo. 


\section{Reconocimiento}

Agradecimiento a la Universidad Autónoma de Querétaro, Facultad de Ingeniería.

\section{Referencias}

Block, D., Fuenlabrada, I., Balbuena, H., \& Ortega, L. (1997). Lo que cuentan las cuentas de multiplicar y dividir. SEP.

Block, D., Mendoza, T., \& Ramírez, M. (2015). ¿Al doble le toca el doble? La enseñanza de la proporcionalidad en la educación básica. SM Editorial.

Broitman, C. (2000). Las operaciones en el primer ciclo. Aportes para trabajo en el aula. Ediciones Novedades Educativas.

Brousseau, G. (2000). Educación y didáctica de las matemáticas. Educación Matemática, $12, \quad 5-38 . \quad \mathrm{http} / / / \mathrm{www} \cdot$ revista-educacionmatematica.org.mx/descargas/Vol12/1/03Brousseau.pdf

Chalmers, C. (2018). Robotics and computational thinking in primary school. International Journal of Child-Computer Interaction, 17, 93-100. https://doi.org/10.1016/j.ijcci.2018.06.005

Di Lieto, M. C., Inguaggiato, E., Castro, E., Cecchi, F., Cioni, G., Dell'Omo, M., Laschi, C., Pecini, C., Santerini, G., Sgandurra, G., \& Dario, P. (2017). Educational Robotics intervention on Executive Functions in preschool children: A pilot study. Computers in Human Behavior, 71, 16-23. https://doi.org/10.1016/j.chb.2017.01.018

Echeverría-Anaya, C. (2019). La resolución de problemas y su incidencia en la enseñanza del pensamiento numérico en los estudiantes de $3^{\circ}$ de la Institución Educativa de Yati. Revista Tecnológica-Educativa Docentes $\quad 2.0 ., \quad 7 \quad$ (2), 123-131. https://ojs.docentes20.com/index.php/revistadocentes20/article/view/19

Fregona, D. (2013). Una propuesta de análisis para la preparación y gestión de clases de matemática. Cuadernos de Educación, (11), 1-16.

Hernández, R., Fernández, C., \& Baptista, P. (2010). Metodología de la Investigación (Quinta). McGrawHill.

Mataric, M. J. (2004). Robotics education for all ages. Proceedings, AAAI Spring Symposium on Accessible, Hands-on AI and Robotics Education. https://www.aaai.org/Papers/Symposia/Spring/2004/SS04-01/SS04-01-004.pdf

Mochón-Cohen, S. (2012). Enseñanza del razonamiento proporcional y alternativas para el manejo de la regla de tres. Educación Matemática, $\quad 24 \quad$ (1), 133-157. http://www.scielo.org.mx/scielo.php?script=sci_arttext\&pid=S1665 $-58262012000100006 \& \operatorname{lng}=$ es\&tlng=es.

OCDE. (2017). Marco de Evaluación y de Análisis de PISA para el Desarrollo: Lectura, matemáticas y ciencias. Versión preliminar. OECD Publishing.

OCDE. (2019). PISA 2018 Results (Volume I). OECD. https://doi.org/10.1787/5f07c754-en

Block, D., Fuenlabrada, I., Balbuena, H., \& Ortega, L. (1997). Lo que cuentan las cuentas de multiplicar y dividir. SEP.

Block, D., Mendoza, T., \& Ramírez, M. (2015). ¿Al doble le toca el doble? La enseñanza de la proporcionalidad en la educación básica. SM Editorial.

Broitman, C. (2000). Las operaciones en el primer ciclo. Aportes para trabajo en el aula. Ediciones Novedades Educativas.
Brousseau, G. (2000). Educación y didáctica de las matemáticas. Educación Matemática, 12, 5-38. http://www.revista-educacionmatematica.org.mx/descargas/Vol12/1/03Brousseau.pdf

Chalmers, C. (2018). Robotics and computational thinking in primary school. International Journal of Child-Computer Interaction, 17, 93100. https://doi.org/10.1016/j.ijcci.2018.06.005

Di Lieto, M. C., Inguaggiato, E., Castro, E., Cecchi, F., Cioni, G., Dell'Omo, M., Laschi, C., Pecini, C., Santerini, G., Sgandurra, G., \& Dario, P. (2017). Educational Robotics intervention on Executive Functions in preschool children: A pilot study. Computers in Human Behavior, 71 , 16-23. https://doi.org/10.1016/j.chb.2017.01.018

Echeverría-Anaya, C. (2019). La resolución de problemas y su incidencia en la enseñanza del pensamiento numérico en los estudiantes de $3^{\circ}$ de la Institución Educativa de Yati. Revista Tecnológica-Educativa $\begin{array}{lllll}\text { Docentes } & 2.0 . & 7 & \text { (2), }\end{array}$ https://ojs.docentes20.com/index.php/revistadocentes20/article/view/19

Fregona, D. (2013). Una propuesta de análisis para la preparación y gestión de clases de matemática. Cuadernos de Educación, (11), 1-16.

Hernández, R., Fernández, C., \& Baptista, P. (2010). Metodología de la Investigación (Quinta). McGrawHill.

Mataric, M. J. (2004). Robotics education for all ages. Proceedings, AAAI Spring Symposium on Accessible, Hands-on AI and Robotics Education. https://www.aaai.org/Papers/Symposia/Spring/2004/SS 04-01/SS04-01-004.pdf

Mochón-Cohen, S. (2012). Enseñanza del razonamiento proporcional y alternativas para el manejo de la regla de tres. Educación Matemática, $\quad 24 \quad$ (1), 133-157. http://www.scielo.org.mx/scielo.php?script=sci_arttext\&pid=S1665 $-58262012000100006 \& \operatorname{lng}=\mathrm{es} \& \ln \mathrm{g}=\mathrm{es}$.

OCDE. (2017). Marco de Evaluación y de Análisis de PISA para el Desarrollo: Lectura, matemáticas y ciencias. Versión preliminar. OECD Publishing.

OCDE. (2019). PISA 2018 Results (Volume I). OECD. https://doi.org/10.1787/5f07c754-en

Papert, S., \& Harel, I. (2002). Situar el construccionismo (pp. 1-20). INCAE.

http://web.media.mit.edu/ calla/web_comunidad/Readings/situar_el construccionismo.pdf

Ruiz-Velasco, E., García, J., \& Rosas, L. (2010). Robótica pedagógica virtual para la inteligencia colectiva (pp. 1-17). Universidad Nacional Autónoma de México.

SEP. (2011). Programas de estudio 2011. Guía para el maestro. Educación Básica. Secretaría de Educación Pública http://basica.sep.gob.mx/dgdc/sitio/pdf/inicio/matlinea/2011/segund o_grado.pdf

Torres-Chávez, B. del C. (2019). La Resolución De Problemas Matemáticos Y Su Incidencia En El Aprendizaje Del Pensamiento Aleatorio De Los Estudiantes De Quinto Grado De Educación Básica De La Institución Educativa Liceo Joaquín F. Vélez. Revista Tecnológica-

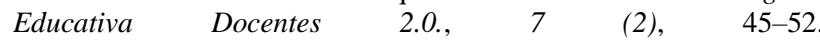
https://ojs.docentes20.com/index.php/revistadocentes20/article/view/10

Vargas, H. el at. (2019). Robótica educativa: Un nuevo entorno interactivo y sostenible de aprendizaje en la educación básica. Revista Tecnológica-Educativa Docentes 2.0., 7 (1), 51-64. https://ojs.docentes20.com/index.php/revistadocentes20/article/view/26 